\title{
FATORES QUE INFLUENCIARAM A EVOLUÇÃO DE 206 PACIENTES COM TRAUMATISMO CRANIENCEFÁLICO GRAVE
}

\author{
Venâncio Pereira Dantas Filho ${ }^{1}$, Antonio Luis Eiras Falcão², \\ Luis Antonio da Costa Sardinha², José Jorge Facure ${ }^{1}$, \\ Sebastião Araújo², Renato G.G. Terzi ${ }^{1,2}$
}

\begin{abstract}
RESUM 0 - A busca de fatores prognósticos para o traumatismo craniencefálico (TCE) tem sido alvo de muitos estudos nas últimas décadas. $A$ identificação de indicadores consistentes da evolução destes pacientes tem representado um grande desafio e sua utilidade considerada evidente tanto para orientar o tratamento, quanto para a estimativa do resultado final. Baseados numa casuística de 206 pacientes com TCE grave (8 pontos ou menos pela Escala de Coma de Glasgow - ECG), estudamos a influência de vários fatores sobre a evolução dos pacientes. A gravidade inicial medida pela ECG, a presença de hipertensão intracraniana (níveis acima de 20 $\mathrm{mmHg}$ ), 0 tipo de lesão intracraniana e a presença de hipoxia, hipotensão arterial e a associação de hipóxia e hipotensão arterial tiveram influência significativa sobre a evolução dos pacientes. A presença de politraumatismo (pelo menos dois sítios de lesão além do TCE) e a idade (acima e abaixo de 40 anos) não influenciaram significativamente a evolução dos pacientes desta casuística.

PALAVRAS-CHAVE: traumatismo craniencefálico, pressão intracraniana, hipóxia, hipotensão arterial, politraumatismo, idade, prognóstico.
\end{abstract}

\begin{abstract}
Relevant factors in 206 patients with severe head injury
ABSTRACT - The search for head injury prognostic factors has been intense in the last decades. The importance of identification of these factors has been also recognised to treatment orientation and results estimatives. Based on 206 severe head injuried patients series, we analized the influence of factors over the outcome. The initial severity by Glasgow coma scale, the presence of intracranial hypertension (over $20 \mathrm{mmHg}$ ), the type of intracranial lesion and the presence of hypoxia, systemic hypotension or both, significantly influenced the results. The presence of multiple traumas (at least two sites of lesion over head injury), as age, did not influence the final results in this series.
\end{abstract}

KEY WORDS: head injury, intracranial pressure, hypoxia, hypotension, multiple trauma, age, outcome.

0 traumatismo craniencefálico (TCE) grave permanece um sério problema em nosso meio. Uma razoável padronização da condução desses pacientes tem sido conseguida com a introdução de protocolos de tratamento tanto no Brasil como em outros países ${ }^{1,2}$. Esta padronização favorece a comparação de casuísticas e conclusões melhor embasadas em evidências.

Estudando uma casuística de 206 pacientes tratados de forma padronizada, analisamos a influência de fatores na evolução final do tratamento.

MÉTODO
Foram analisados 206 pacientes vítimas de TCE grave, definido como aqueles com 8 pontos ou menos na Escala de Coma de Glasgow (ECG) após reanimação inicial, tratados na Unidade de Terapia Intensiva (UTI) do Hospital das Clínicas da Universidade Estadual de Campinas (HC-UNICAM P) segundo um protocolo padronizado, orientado pela monitorização da pressão intracraniana (PIC). Este estudo recebeu parecer favorável (n. 507/2000) da Comissão de Ética Médica do HC - UNICAM P. Os detalhes técnicos foram descritos previamente ${ }^{2-}$ ${ }^{4}$. Desses pacientes, 166 eram do sexo masculino $(80,58 \%)$ e 40 do feminino $(19,42 \%)$. A média de idade foi 29,21 anos, com mediana de 25 anos, variando de um mínimo de 11 a um máximo de 74 anos, com $168(81,55 \%)$ pacientes apresentando idade menor ou igual a

1Disciplina de Neurocirurgia do Departamento de Neurologia e Neurocirurgia da Faculdade de Ciências Médicas da Universidade Estadual de Campinas, Campinas SP, Brasil (UNICAM P); ${ }^{2}$ Unidade de Terapia Intensiva do Hospital das Clínicas da UNICAM P

Recebido 7 Julho 2003, recebido na forma final 30 Setembro 2003. Aceito 10 Novembro 2003.

Dr. Venâncio Pereira Dantas Filho - Avenida Estados Unidos 219 - 13036-390 Campinas SP - Brasil. E-mail: venancio@hc.unicamp.br 
40 anos e $38(18,45 \%)$ com idade acima de 40 anos.

As causas do TCE foram os acidentes de trânsito (automóvel, motocicleta e atropelamentos) em 147 casos (71,36\%), as quedas em 37 casos $(17,96 \%$ ) e os traumas diretos sobre o segmento cefálico por queda de objetos ou agressões em 15 casos $(7,28)$. A causa do TCE não foi conhecida em 7 pacientes (3,40\%). Foram excluídos os pacientes vítimas de ferimentos cranianos por projétil de arma de fogo.

Foram consideradas lesões associadas ao TCE os traumatismos em face, membros, tórax, abdômen e coluna, que necessitaram de tratamento específico. Foram considerados politraumatizados 49 pacientes $(23,79 \%)$ com pelo menos dois sítios de lesão além do TCE.

Foram considerados hipóxicos os pacientes que apresentaram evidentes sinais clínicos (cianose) ou intercorrências associadas a hipóxia na admissão (pacientes transportados por longas distâncias sem intubação e suporte ventilatório, ou com suporte ventilatório inadequado, além de crises convulsivas prévias). Foram considerados hipotensos os pacientes que apresentaram na admissão pressão arterial sistólica menor que $90 \mathrm{~mm} \mathrm{Hg}$.

Após os procedimentos de reanimação (intubação traqueal e ventilação adequada, além de reposição volêmica) no atendimento inicial no pronto-socorro (PS), os pacientes foram considerados graves ( 6 a 8 pontos na ECG) ou muito graves ( 3 a 5 pontos na ECG).

Todos os pacientes foram submetidos a tomografia computadorizada de crânio (TC), sendo em seguida encaminhados para internação na UTI. As lesões intracranianas identificadas na TC foram classificadas segundo critérios de Marshall e col. ${ }^{5}$ em: lesão difusa I (sem patologia visível à TC); lesão difusa II (cisternas presentes, desvio da linha média menor que $5 \mathrm{~mm}$, lesões expansivas menores que $25 \mathrm{ml}$, podendo estar presentes corpos estranhos ou fragmentos ósseos); lesão difusa III - inchaço (cisternas comprimidas, desvio da linha média menor que $5 \mathrm{~mm}$, lesões expansivas menores que $25 \mathrm{ml}$ ); lesão difusa IV - desvio (desvio da linha média maior que $5 \mathrm{~mm}$, lesões expansivas menores que $25 \mathrm{ml}$ ); lesão focal operada (qualquer lesão expansiva tratada cirurgicamente); lesão focal não operada (qualquer lesão expansiva maior que $25 \mathrm{ml}$ não tratada cirurgicamente).

Uma vez internados na UTI e corrigidas todas as alterações sistêmicas possíveis, os pacientes foram mantidos em tratamento geral para o TCE grave, que compreendeu as seguintes medidas ${ }^{2,3,6}: 1$ -
Decúbito elevado, a $30^{\circ}$. 2- Sedação com tionembutal ou com fentanil associado a midazolan. 3- Ventilação mêcanica com os parâmetros do ventilador para uma $\mathrm{PaCO}_{2}$ entre 30 e $35 \mathrm{~mm} \mathrm{Hg}$.

Todos os pacientes foram submetidos a monitorização contínua da $\mathrm{PIC}^{2}$ sendo considerados aceitáveis os níveis até $20 \mathrm{~mm} \mathrm{Hg}$, não necessitando de tratamento específico. Nestes pacientes foram mantidas as medidas terapêuticas gerais e uma vez mantidos esses níveis de PIC por pelo menos 48 hs, foram iniciados procedimentos de retirada da sedação, ventilação mecânica e monitorização da PIC.

Nos pacientes em que a PIC se manteve acima de $20 \mathrm{~mm} \mathrm{Hg}$ foi iniciado tratamento específico, progressivo e cumulativo para a hipertensão intracraniana $(\mathrm{HIC})^{2,3,6}$. Cada nova conduta só foi iniciada após a verificação da refratariedade da PIC à conduta anterior: 1Hiperventilação com níveis de $\mathrm{PaCO}_{2}$ abaixo de $30 \mathrm{~mm} \mathrm{Hg}$. 2- Manitol a $20 \%$ endovenoso, em bolo e infusão rápida, na dose de $1 \mathrm{~g} / \mathrm{kg}$. 3-Aumento progressivo da sedação. 0 tratamento específico para a HIC foi otimizado, na maioria dos pacientes, através da medida intermitente da extração cerebral de $\mathrm{O}_{2}$ (diferença entre a saturação da oxi-hemoglobina arterial e do sangue venoso jugular, colhido por meio de cateter posicionado no bulbo da veia jugular interna) $)^{6,7}$.

Foram realizadas análises comparativas entre grupos de pacientes divididos de acordo com as variáveis. Foram realizados o teste do quiquadrado e o teste exato de Fisher conforme o indicado. Foi adotado o nível de significância de 5\% para as análises realizadas.

\section{RESULTADOS}

Faleceram 75 pacientes (36,40\%), 38 (18,45\%) tiveram alta não respondendo a comandos verbais simples (ECG de 8 pontos ou menos), $45(21,85 \%)$ pacientes respondiam a comando verbal simples (ECG de 9 a 12 pontos) e 48 (23,30\%) respondiam a comandos verbais complexos (ECG de 13 a 15 pontos) no momento da alta hospitalar.

A Tabela 1 mostra o resultado da análise entre variáveis (ECG menor ou igual a 5 pontos, presença de hipóxia, hipotensão arterial ou ambas, PIC maior que $20 \mathrm{mmHg}$, idade maior que 40 anos e presença de politraumatismo) e o resultado na alta hospitalar. A Tabela 2 mostra o resultado da análise entre o tipo de lesão intracraniana e o resultado.

A presença de hipóxia, hipotensão arterial e sua associação

Tabela 1. Resultado do tratamento de acordo com ECG de 3-5 pontos, presença de hipóxia, hipotensão arterial ou ambos, PIC acima de 20 $\mathrm{mmHg}$, idade acima de 40 anos e presença de politraumatismo.

\begin{tabular}{lccccc}
\hline & óbito (75) & $3-8(38)$ & $9-12(45)$ & $13-15(48)$ & $p$ \\
\hline ECG 3-5 & 46 & 23 & 18 & 3 & $<0,001$ \\
com hipóxia & 43 & 17 & 13 & 8 & $<0,001$ \\
com hipotensão & 25 & 6 & 4 & 4 & $<0,001$ \\
Com hipoxia+hipotensão & 17 & 1 & 2 & 2 & $<0,001$ \\
PIC > 20 mmHg & 71 & 25 & 23 & 18 & $<0,001$ \\
$>40$ anos & 15 & 7 & 10 & 6 & NS \\
com politrauma & 18 & 7 & 12 & 12 & NS \\
\hline
\end{tabular}

NS, não significante 
entre pacientes com e sem politraumatismos está demonstrada na tabela 3. A mortalidade entre pacientes politraumatizados com hipóxia, hipotensão arterial e sua associação encontrase na Tabela 4.

Apresentaram pelo menos uma complicação sistêmica que merecesse tratamento 172 pacientes $(83,50 \%)$. Foram consideradas complicações sistêmicas os distúrbios eletrolíticos (hipo ou hipernatremia e hipo ou hipercalemia) presentes em 130 casos $(63,11 \%)$, broncopneumonia em 119 casos $(57,77 \%)$, poliúria (volume urinário acima de $300 \mathrm{ml} / \mathrm{h}$ ) em 32 (15,53\%), infecção urinária em 11 (5,34\%), sepse independente do foco em 10 casos $(4,85 \%)$, sinusite em $6(2,91 \%)$ e sangramento gastrointestinal em 3 casos (1,46\%).

A distribuição da gravidade, níveis de PIC, presença de hipóxia, hipotensão arterial ou sua associação presença de complicações sistêmicas entre pacientes abaixo e acima de 40 anos é mostrado na Tabela 5.

\section{DISCUSSÃO}

A gravidade clínico-neurológica inicial, medida através da ECG, influenciou significativamente a evolução dos pacientes desta casuística ( $p<0,001$ ) (Tabela 1), ficando demonstrado, portanto, que a manifestação clínica inicial é um forte indicador da gravidade das lesões primárias e secundárias associadas ao TCE. A ECG classicamente tem se mostrado bastante útil, não só para a padronização e comparação da avaliação da gravidade do envolvimento neurológico nas casuísticas, mas também como forte indicador prognóstico nas doenças traumáticas e não-traumáticas ${ }^{8}$.

A presença de hipóxia estimada por critérios clínicos certamente identifica pacientes com hipóxia mais severa, não identificando aqueles com graus menos profundos de hipóxia9 Mesmo assim, 81 pacientes desta casuística (40\% do total) encontravam-se em hipóxia na sua admissão. Segundo estes critérios, metade destes (43 pacientes) evoluiu para o óbito, sendo essa influência bastante significativa $(p<0,001)$ (Tabela 1).

Praticamente $20 \%$ (39 casos) dos pacientes apresentaram pressão arterial sistólica abaixo de $90 \mathrm{~mm} \mathrm{Hg}$ no momento da admissão, tendo dois terços destes $(64,10 \%)$, ou seja 25 pacientes, evoluído para o óbito ( $p<0,001$ ) (Tabela 1). A associação de hipóxia e hipotensão arterial esteve presente em $10 \%$ dos pacientes ( 22 casos) e, quando presente, resultou em óbito em quase 80\% (17 casos) ( $p<0,001$ ) (Tabela 1 ). Chesnut ${ }^{10}$ referiu que a associação de hipóxia com hipotensão arterial levou à uma mortalidade de $75 \%$ entre pacientes com TCE grave. Miller e col. ${ }^{11}$ relataram uma incidência de 30\% de hipóxia (documentada por gasometria) e 13\% de hipotensão

Tabela 2. Influência do tipo de lesão intracraniana sobre o resultado.

\begin{tabular}{lcccc}
\hline Tipo de lesão & óbito & $3-8$ & $9-12$ & $13-15$ \\
\hline difusa I & - & 2 & 5 & 8 \\
difusa II & 18 & 15 & 13 & 17 \\
difusa III & 18 & 6 & 3 & 6 \\
difusa IV & 9 & 2 & 2 & - \\
focal operada & 22 & 13 & 20 & 17 \\
focal não op. & 8 & - & 2 & - \\
\hline
\end{tabular}

$p<0,001$ - Qui - quadrado $=25,720$ (8 graus de liberdade) - foram agrupados os resultados 9-12 e 13-15 e as lesões focais

Tabela 3. Distribuição de pacientes com hipóxia, hipotensão arterial ou ambos entre pacientes com e sem politraumatismo.

\begin{tabular}{lccc}
\hline & $\begin{array}{c}\text { sem } \\
\text { politrauma } \\
(157)\end{array}$ & $\begin{array}{c}\text { com } \\
\text { politrauma } \\
(49)\end{array}$ & $p$ \\
\hline com hipóxia & 62 & 19 & NS \\
com hipotensão & 20 & 19 & $<0,001$ \\
com hipóxia+hipotensão & 10 & 12 & $<0,001$ \\
\hline NS, não significante. & & & $p$ \\
Tabela 4. Mortalidade entre pacientes politraumatizados com hipóxia, hipotensão arterial ou sua \\
associação. & & & $<0,05$ \\
\hline & óbito (18) & sobrevida (31) & $<0,05$ \\
com hipóxia (19) & 11 & 8 & $<0,05$ \\
Com hipotensão (19) & 11 & 8 & \\
com hipóxia+hipotensão (12) & 8 & 4 & \\
\hline
\end{tabular}


Tabela 5. Distribuição de ECG de 3-5 pontos, PIC acima de $20 \mathrm{mmHg}$, presença de complicações sistêmicas, presença de hipóxia, hipotensão arterial e sua associação entre pacientes acima e abaixo de 40 anos.

\begin{tabular}{lccc}
\hline & $\leq 40$ anos & $>40$ anos & $p$ \\
\hline ECG 3-5 & 78 & 12 & NS \\
PIC $>20 \mathrm{~mm} \mathrm{Hg}$ & 111 & 26 & NS \\
com complicações & 140 & 32 & NS \\
com hipóxia & 70 & 11 & NS \\
com hipotensão & 34 & 5 & NS \\
com hipóxia+hipotensão & 19 & 3 & NS \\
\hline
\end{tabular}

NS, não significante.

arterial em uma casuística de 100 pacientes com TCE grave associados a aumento significativo da morbidade e mortalidade, estando a hipotensão associada a múltiplos traumas e a hipóxia não. A influência negativa sobre os resultados, proporcionada pela presença de hipóxia, hipotensão arterial sistêmica ou pela associação desses eventos, pode ser também evidenciada quando observamos que politraumatismos associados aos mesmos fatores tiveram mortalidade significativamente maior ( $p<0,05$ para cada uma dessas situações) (Tabela 4). Estes resultados confirmam as afirmações de Miller e col. ${ }^{11,12}$ e Becker e col..$^{13}$ que chamaram a atenção para a influência indireta dos politraumatismos sobre a evolução do TCE.

Os politraumatismos isoladamente não influenciaram significativamente o resultado final desta casuística (Tabela 1), como também demonstrado por Miller e col. ${ }^{12}$ e Gennarelli e col. ${ }^{14}$. Entretanto, levaram a uma significativa maior incidência de hipotensão arterial e da associação entre hipóxia e hipotensão arterial ( $p<0,001$ em ambas situações) (Tabela 3). Estas observações estão de acordo com as afirmações de Miller e col. ${ }^{12}$ e Becker e col. ${ }^{13}$.

A hipóxia isoladamente distribuiu-se sem diferenças significativas entre os pacientes com ou sem politraumatismos (Tabela 3). A pesar de freqüentemente associar-se às lesões de face e tórax nos politraumatizados, não necessariamente precisa dessas lesões para se instalar. Miller e col..$^{12}$ da mesma forma observaram distribuição homogênea de hipóxia entre pacientes politraumatizados e não politraumatizados.

0 tipo de lesão intracraniana, segundo critérios de M arshall e col. ${ }^{5}$ influenciou significativamente a evolução dos pacientes desta casuística $(p<0,001)$ (Tabela 2). Nossas observações confirmam a utilidade desta classificação, baseada unicamente no aspecto da TC inicial, para inferência prognóstica e identificação de pacientes com maior risco de piora clínica evolutiva.

A significativa influência dos níveis de PIC sobre o resultado
( $p<0,001$ ) (Tabela 1), com predomínio de maus resultados (ECGI de 8 ou menos eóbitos) relacionados a níveis de PIC acima de $20 \mathrm{~mm} \mathrm{Hg}$, demonstra a importância da identificação da HIC o mais precocemente possível. Todos os esforços devem ser voltados para seu controle, tanto por meio de medidas cirúrgicas quanto medicamentosas ${ }^{1-4}$.

A idade não influenciou significativamente a evolução dos pacientes (Tabela 1), apesar da tradicional expectativa de melhor evolução entre os mais jovens e de pior evolução entre os mais velhos ${ }^{15}$. Da mesma forma não foram significativamente diferentes as distribuições da gravidade (ECG 3-5), níveis de PIC, incidência de complicações extracranianas, hipóxia, hipotensão arterial e associação de hipóxia e hipotensão arterial entre os pacientes agrupados acima e abaixo de 40 anos de idade (Tabela 5).

Apesar de não significativa, a distribuição de pacientes muito graves e graves entre os grupos acima e abaixo de 40 anos mostrou-se bastante desproporcional, com $46,42 \%$ dos pacientes mais jovens muito graves (78 pacientes de 168) e $31,57 \%$ dos pacientes muito graves no grupo acima de 40 anos (12 pacientes de 38) (Tabela 5). Foram observadas também apenas 3 pacientes muito graves de 13 acima de 50 anos e nenhum muito grave entre 6 pacientes acima dos 60 anos. Como a gravidade foi desproporcionalmente menor entre os mais velhos, fica mais evidente porque não houve uma influência negativa da idade sobre o resultado.

Essa desproporcionalidade entre as faixas etárias e a distribuição da gravidade tem como provável fator relacionado a grande incidência de traumas graves (incluindo o TCE) entre a população jovem mais exposta. Outro provável fator relacionado seria a grande demanda por insuficientes leitos intensivos especializados, tanto no HC-UNICAM P quanto na totalidade dos hospitais públicos em nosso país. Esta situação levaria a diminuição proporcional de pacientes mais velhos e mais graves na utilização de leitos intensivos, privilegiando pacientes mais jovens. Marino $\mathrm{Jr}^{16}$ relatou a existência de 
menos de 600 leitos especializados em doenças neurológicas em todo o Estado de São Paulo, suficientes para atender apenas $3 \%$ da demanda. Relatou também que 80 a $90 \%$ dos pacientes do pronto-socorro do Hospital das Clínicas de São Paulo são pacientes neurológicos (a maioria com TCE), com índices de ocupação de $90 \%$ de leitos da Clínica Neurológica daquele hospital, na maioria das vezes sem o benefício de uma terapia intensiva. Dantas e M oretti-Branchini ${ }^{17}$ relataram que $55,18 \%$ dos pacientes na unidade de observação do PS do HC - UNICAMP eram portadores de doenças neurológicas, com média de idade de 51,05 anos (66,81\% com mais de 40 anos), entre 232 pacientes analisados entre março de 1996 e junho de 1998. Castelar ${ }^{18}$ relatou uma diminuição progressiva da oferta de leitos públicos no Brasil na década de 80 , reconhecendo que nosso país, na área da saúde, enfrenta um processo de crise, com escassez de recursos financeiros, que se torna ainda mais dramática diante das aceleradas transformações tecnológicas e da crescente demanda de melhores produtos e serviços. Apontou como dramática a situação de carência de leitos especializados, principalmente terapia intensiva de adultos, pediátrica e neonatal, unidades coronarianas, queimados e neurocirurgia, nesta ordem.

Um terceiro e menos provável fator relacionado a essa situação seria o fato de pacientes com mais idade não sobreviverem tempo suficiente para receberem atendimento médico, sendo atendidos apenas os pacientes menos graves, ou seja, os traumatizados de crânio sobreviventes. Esta hipótese fica praticamente afastada quando entendemos o TCE como um espectro contínuo de gravidade ${ }^{19}$. Assim, os pacientes idosos mais graves morreriam antes do atendimento, pela piora adicional durante a fase pré-hospitalar, como também outros pacientes idosos menos graves da mesma forma piorariam durante esta fase, apresentando-se muito graves no momento do atendimento inicial. Pelo contrário, Kraus e col. ${ }^{20}$ relataram um tempo de sobrevida maior entre pacientes mais velhos que morreram vítimas de TCE, quando comparados a pacientes mais jovens, sendo essa situação provavelmente relacionada a diferenças, dependentes da idade, na incidência e evolução de complicações associadas ao trauma.

\section{CONCLUSÕES}

A análise desta casuística confirma a influência de fatores clássicos como a gravidade, hipertensão intracraniana e tipo de lesão intracraniana sobre a evolução de pacientes com TCE grave. A influência deletéria da presença de hipóxia, hipotensão arterial ou ambas, na admissão, ressalta a importância da prevenção a todo custo desses eventos no atendimento préhospitalar de pacientes traumatizados. A ausência de influência significativa da idade sobre a evolução dos pacientes nesta casuística levanta uma importante e ampla discussão sobre 0 atendimento de idosos em nosso país, sugerindo que problemas de financiamento do atendimento público da saúde possam estar tendo impacto direto sobre o tratamento especializado desses pacientes, vítimas ou não de traumatismo craniano. 


\section{REFERÊNCIAS}

1. Kelly DF, Doberstein C, Becker DP. General principles of head injury management. In: Narayan RK, Wilberger JE Jr, Povlishock JT (EDS). Neurotrauma. New York: McGraw-Hill, 1996:71-101.

2. Dantas VP Filho, Falcão ALE, Sardinha LAC, Facure JJ, Araújo S, Terzi RGG. Aspectos técnicos da monitorização da pressão intracraniana pelo método subaracnóideo no traumatismo craniencefálico grave. Arq Neuropsiquiatr 2001;59:895-900.

3. Dantas VP Filho. Aspectos técnicos da monitorização da pressão intracraniana pelo método subaracnóideo e análise dos fatores que influenciaram a evolução de 206 pacientes com traumatismo craniencefálico grave. Tese, UNICAMP, Campinas:1999.

4. Falcão ALE, Dantas VP Filho, Saraiva JFK, Sardinha LAC, Araújo S, Terzi RGG. Bedside ICP monitoring in head trauma patients by using a simple, safe and low cost methodology. Clin Intens Care 1992;3(Suppl):112.

5. Marshall LF, Marshall SB, Klauber MR, et al. A new classification of head injury based on computerized tomography. J Neurosurg 1991;75(Suppl):S14-S20.

6. Falcão ALE, Dantas VP Filho, Sardinha LAC, et al. Highlighting intracranial pressure monitoring in patients with severe acute brain trauma. Arq Neuropsiquiatr 1995;53:390-394.

7. Cruz J. Relevância da otimização ventilatória em hipertensão intracraniana aguda: uma abordagem clínica-fisiológica-terapêutica. Arq Neuropsiquiatr 1995;53:131-140.

8. Marion DW. Outcome from severe head injury. In Narayan RK, Wilberger JL Jr, Povlishock JT (EDS). Neurotrauma. New York: McGraw-Hill, 1996:767-777.

9. Luerssen TG, Marshall LF. The medical management of head injury. In Vinken PJ, Bruyn GW, Klawans HL, Braakman R (EDS). Handbook of clinical neurology: head injury. Amsterdam: Elservier, 1990:207-247.
10. Chesnut RM. Medical complications of the head injury patients. In Cooper PR (ed). Head injury, 3.Ed. Baltimore: Williams \& Wilkins, 1993:459-501

11. Miller JD, Sweet RC, Narayan R, Becker DP. Early insults to the injuried brain. JAMA 1978;240:439-442.

12. Miller JD, Butterworth JF, Gudeman SK, et al. Further experience in the management of severe head injury. J Neurosurg 1981;54:289-299.

13. Becker DP, Gade GF, Young HF, Feuerman TF. Diagnosis and treatment of head injury in adults. In Youmans JR (ed). Neurological surgery 3.Ed. Philadelphia: Saunders, 1990:2017-2148.

14. Gennarelli TA, Champion HR, Sacco WJ, Copes WS, Alves WM. Mortality of patients with head injury and extracranial injury treated in trauma centers. J Trauma 1989;29:1193-1201.

15. Jane JA, Francel PC. Age and outcome of head injury. In Narayan RK, Wilberger JE Jr, Povlishock JT (EDS). Neurotrauma. New York: McGrawHill, 1996:793-804.

16. Marino R Jr. A década do cérebro. Consultório Médico 1991;6:37-38.

17. Dantas SRPE, Moretti-Branchini ML. Impact of antibiotic-resistant pathogens colonizing the respiratory secretions of patients in na extended-care area of Emergency Departament. Infect Control Hosp Epidemiol 2003;24:351-355.

18. Castelar RM. O hospital no Brasil. In Castelar RM, Modelet P, Grabois V (EDS). Gestão hospitalar: um desafio para o hospital brasileiro. Rennes: Éditions ENSP, 1995:38-49.

19. Rimel RW, Giordani B, Barth JT, Jane JA. Moderate head injury: completing the clinical spectrum of brain trauma. Neurosurgery 1982;11:344-351.

20. Kraus JF, Conroy C, Cox P, Ramstein K, Fife D. Survival times and case fatality rates of brain injuried patients. J Neurosurg 1985;63:537-543. 